\title{
Splinting does not affect the oral tissues surrounding the implants
}

\begin{abstract}
Naert I, Gizani S, Vuylsteke, Van Steenberghe D. A 5-year randomised clinical trial on the influence of splinted and unsplinted oral implants in the mandibular overdenture therapy. Part 1. Peri-implant outcome. Clin Oral Impl Res
\end{abstract} 1998; 9: 170-177

Objectives To investigate treatment outcome of splinted and unsplinted implants retaining mandibular hinging overdenture.

Design 5-year prospective randomised-controlled-trial (RCT)

Setting Prosthetic Department of Dental School in Belgium.

Patients 36 edentulous patients ( 17 male, 19 female) with a Mean age of 63 yrs (range 36-8 5 years). Exclusion criteria were, insufficient bone volume to harbour at least $210 \mathrm{~mm}$ implants, Angle class II jaw relationship, physiological problems for accepting dentures, gagging reflexes, less than 1 year of edentulism in mandible, absence of a maxillary complete denture and administrative or physical considerations that would seriously affect the surgical procedure or follow-up.

Intervention Each patient was provided with 2 screw shaped c.p. titanium implants (Branemark system). 3-5 months later transmucosal abutments were placed. The patients were randomised to 3 groups with different retention systems, (a bar group, a magnet group and a ball group) for attachment of the overdenture. Patients received regular follow-up for $60 \mathrm{mths}$.

\begin{abstract}
Results 5 patients failed to complete all the follow-up visits ( 3 in Bar group, 1 Magnet, 1 Ball ). None of the loaded implants failed. 1 implant was replaced at abutment surgery and survived. Of the periimplant outcomes considered ( plaque levels, mucosal bleeding, pocket depth, attachment level and marginal bone level) the only significant finding was a higher level of plaque in the magnet group compared to the ball group. No differences in plaque levels were noted between the bar and ball or between the bar and magnet groups.
\end{abstract}

Conclusions The peri-implant outcome is hardly any difference between splinted and unsplinted implants, Plaques levels a slightly higher on abutments fitted with magnets. There was no correlation between bleeding on probing and marginal bone loss. Marginal bone loss and attachment loss were not statistically different at year 5. The bone-implant interface rigidity was equal between the groups and increased over time.

Address I Naert, Department of Prosthetic Dentistry, BIOMAT Research Group, School of Dentistry, Oral and Maxillofacial Surgery, Catholic University Leuven, Belgium

\section{Commentary}

A solution to problems with wearing a complete lower denture can be to place 2 implants in the mandible for creating retention for the denture. It is a frequently debated issue if splinting of the 2 implants with a bar is better than mounting an attachments on each implant. Naert and co-workers compared the peri-implant outcome of treatment with mandibular overdentures retained on 2 implants mounted with either a egg-shaped Dolder-bar, openfields magnets or ball attachments.

Only few studies have actually compared different treatment modalities. One of the qualities of the study of Naert and co-workers is that the different retention systems are compared in patients randomly allocated into the 3 groups. The study has a 5-year followup. No implants failed after loading, therefore, none of the retention systems seems to cause overload of the implants. It should be appreciated that the dentures are described as hinging overdentures and, therefore, the load on the implants may be limited. Furthermore, the result may be different in patients with the most poor bone quality.

From the result of this study the 3 retention systems do not seem to have different impacts on the marginal bone loss around the implants.

In general, no difference in the periimplant parameters was observed between the treatment modalities, although implants with magnet reten- tion were harbouring more plaque than the other groups. This finding may be explained by the stated less patient satisfaction with the magnet retention system. Since no important differences in the peri-implant outcome was observed between patients treated with the 3 retention systems, it should be the prosthetic outcome, patient satisfaction, and cost of the treatment that determine which type of retention system is to be preferred.

The prosthetic aspects and the patient satisfaction will be reported in a later paper.

Fleming Isidor

Professor of Prosthetic Dentistry, University of Aarhus, Denmark 